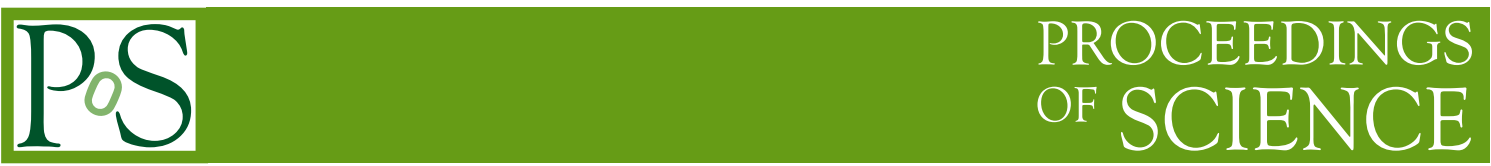

\title{
Searching for clusters using weak lensing
}

\author{
Marco Hetterscheidt *, Thomas Erben and Peter Schneider \\ IAEF Bonn, Germany \\ E-mail: mhetter@astro.uni-bonn.de, terben@astro.uni-bonn.de, \\ petereastro.uni-bonn.de
}

\begin{abstract}
Measuring the distribution and structures of galaxy clusters in a large redshift interval provides crucial information about the history and the large scale structure of the Universe. Weak gravitational lensing provides an opportunity to measure the (projected) mass distribution without any simplifying assumptions, such as hydrostatic equilibrium of the intra-cluster gas and virial equilibrium. Aside from the analysis of already known galaxy clusters, weak lensing techniques can be used to perform a blind search for hitherto unknown mass concentrations. As a consequence a purely mass-selected cluster sample can be obtained which is not biased towards the baryonic content. We briefly describe the method and results of a cluster search using 50 randomly selected $I$-band images which were observed under excellent seeing conditions with the FORS1 camera at the VLT. Several significant peaks are detected in the weak lensing maps, which coincide with overdensities of the light distribution.
\end{abstract}

Baryons in Dark Matter Halos

5-9 October 2004

Novigrad, Croatia

* Many thanks to R. Maoli and L. Van Waerbeke 

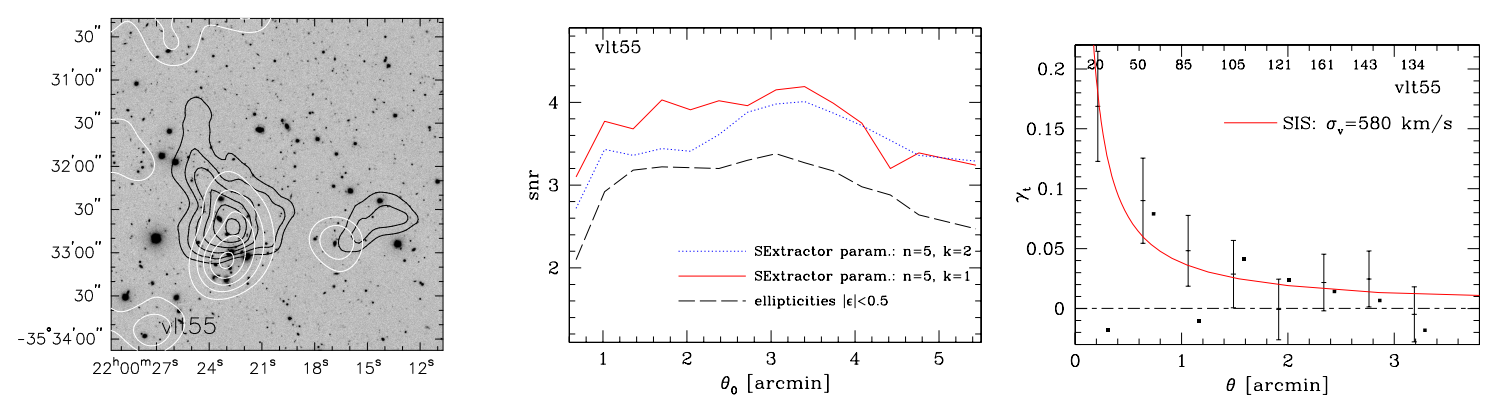

Figure 1: Left panel: magnified image of a VLT-field in which we detected a significant lensing signal (signal-to-noise ratio $s n r \approx 4.3$ ). The $M_{\text {ap }}$ contours are black (snr-values $\left.1.5,2.0,2.5,3.0,3.5,4.0\right)$ and the light distribution contours are white (snr-values 1.0, 2.0, 3.0, 4.0, 5.0). The concentration of luminous galaxies is clearly visible. Middle panel: maximum signal-to-noise ratio of a cluster candidate and its dependence on filter radius, $\theta_{0}$ for different SExtractor parameter settings $(n=5$ contiguous pixels, $k=1 \sigma$ respectively $k=2 \sigma$ above the smoothed sky background). Also shown is a $M_{\text {ap-analysis for galaxy elliptic- }}$ ities $|\varepsilon|<0.5$ (dashed line). Right panel: tangential shape distortion of galaxy images and its dependence on distance, $\theta$, from the maximum of the aperture mass signal. Solid squares: perpendicular shape distortion of galaxy images (not influenced by a gravitational field). Upper numbers: number of background galaxies per bin. We formally fitted an SIS profile with a velocity dispersion of $\sigma_{\mathrm{v}}=580 \mathrm{~km} \mathrm{~s}^{-1}$ (corresponding to $\sim 10{ }^{14} M_{\odot}$, within $500 \mathrm{kpc}$ ), assuming $z_{\text {lens }}=0.3$ and $\left\langle z_{\text {sources }}\right\rangle=1$.

\section{Data}

For the current work we observed 50 uncorrelated fi elds with the FORS1 camera at the VLT at Paranal, chosen such that they are not biased towards overdense or underdense regions. The data was used before for a cosmic shear analysis by Maoli et al. (2001) and have the following characteristics:

1. The area of the CCD chip is $2048 \times 2048$ pixels with a pixel size of $24 \mu \mathrm{m}$, so each fi eld covers $6.8 \times 6.8$, resulting in a total area of $0.64 \mathrm{deg}^{2}$.

2. The observing time for each fi eld was $36 \mathrm{~min}$, and the average seeing was $00^{\prime} 63$, resulting in a limiting magnitude of $I=24.5$, an average number density of galaxies of $n \approx 30 \mathrm{arcmin}^{-2}$ and an average source redshift of $\langle z\rangle \approx 1$.

\section{Method \& Results}

A quantitative way to detect galaxy clusters is provided by the aperture mass $\left(M_{\mathrm{ap}}\right)$ statistics (Schneider 1996). The aperture mass is defi ned as a spatially fi ltered, projected density contrast at a given position $\xi$, and can be determined by means of the observable tangential shape distortion of galaxy images due to the gravitational fi eld of intervening mass concentrations (tangential shear $\left.\gamma_{t}\right)$,

$$
M_{\mathrm{ap}}(\xi)=\int \mathrm{d}^{2} \theta \gamma_{\mathrm{t}}(\theta ; \xi) Q(|\theta-\xi|),
$$

where $Q$ denotes an effective fi lter function which has been introduced by Schirmer (2004). To obtain weak lensing maps, a regular grid is placed over the data fields and the aperture mass is calculated for every grid point $\xi$. In this way we obtain weak lensing maps ( $M_{\mathrm{ap}}$-maps) for every 
VLT-fi eld in which mass concentrations are revealed as peaks in the map. The $M_{\mathrm{ap}}$-maps are compared with light distribution maps. Fig. 1 displays one of the located galaxy cluster candidates with aperture mass and light contours.

The weak lensing maps are contaminated by noise peaks caused by a chance alignment of background galaxies. Therefore we analyze the 50 VLT-fi elds for different $Q$-fi lter radii, $\theta$, and for different source extraction parameter settings (we use $n=5$ contiguous pixels, $k=1 \sigma$ above the smoothed sky background and a more conservative one, $n=5, k=2 \sigma$ ). With the fi rst parameter settings noise sources without shear information are also extracted, which could lower the lensing signal. With the more conservative settings background sources which could contribute to the lensing signal are missed. In both cases the signal-to-noise ratio (snr) should not vary signifi cantly if the signal is due to a real cluster.

A noise peak can also arise if a small number of galaxies with high ellipticities align tangentially by chance. To exclude the possibility that the snr is dominated by such galaxies we restrict the ellipticities to $|\varepsilon|<0.5$ and analyze the candidates once more. Fig.1 displays the snr and its dependence on radius for the different methods.

To obtain a rough estimate of the velocity dispersion $\sigma_{\mathrm{v}}$, and hence the mass of a cluster candidate, we fi t a simple galaxy cluster model (singular isothermal sphere, SIS) to the measured tangential shape distortions,

$$
\gamma_{\mathrm{t}}(\theta)=5^{\prime \prime}\left(\frac{\sigma_{\mathrm{v}}}{600 \mathrm{~km} \mathrm{~s}^{-1}}\right)^{2} \frac{D_{\mathrm{ls}}}{D_{\mathrm{s}}} \frac{1}{\theta},
$$

where $D_{\mathrm{ls}}$ and $D_{\mathrm{s}}$ are the angular diameter distances between lens-source and observer-source, respectively (Fig.1).

We end up with 5 promising cluster candidates with velocity dispersions of roughly $600 \mathrm{~km} \mathrm{~s}^{-1}$. We compare these results with ray tracing through $N$-body simulations (made available by Hamana et al. (2004)) and fi nd that within the measurement uncertainties the observations are in good agreement with predictions from the simulations. The detected candidates need follow-up observations in different fi lters to determine their redshifts and masses.

\section{References}

[1] Schneider, P. 1996b, MNRAS, 313, 524

[2] Maoli, R., Van Waerbeke, L., Mellier, Y., et al. 2001, A\&A, 368, 766

[3] Schirmer, M. 2004, PhD thesis, University of Bonn

[4] Hamana, T., Takada, M. \& Yoshida, N. 2004, MNRAS, 350, 893 